\title{
Opinar sin herir los sentimientos: ¿Cómo enseñar a opinar o valorar de forma atenuada? ${ }^{1}$
}

\author{
DANNY FERNANDO MURILlO LANZA ${ }^{2}$ \\ Universitat de València \\ danny.murillo@uv.es
}

\begin{abstract}
Resumen: Opinar, valorar o emitir un juicio son algunos de los actos de habla más importantes que puede realizar un hablante de cualquier lengua. A través de estos se puede expresar el punto de vista, la percepción, el juicio, la valoración o la evaluación subjetiva que el interlocutor tiene sobre una determinada persona, objeto, situación, comportamiento, etc. En consecuencia, la enseñanza a estudiantes de E/LE de este tipo de actos de habla es igual de esencial. Por ende, el presente artículo tiene como objetivo principal explicar de qué manera se puede opinar o valorar en español de forma atenuada y de qué manera se puede enseñar en el aula de E/LE. Para ello, se propone una unidad didáctica que considera que las opiniones o valoraciones vertidas por jueces de programas de la telerrealidad como Operación Triunfo, MasterChef o La voz pueden ser un material lingüístico atractivo para enseñar a opinar de forma atenuada.
\end{abstract}

Palabras clave: Opinión. Valoración. Atenuación. Programas de la telerrealidad.

Give a opinion without hurting feelings: How to teach to give mitigated opinions or assessments?

Abstract: Opinion, assessment or judgment are some of the most important acts of speech that a speaker of any language can perform. Through these, the point of view, perception, judgment, assessment or subjective evaluation that the interlocutor has about a certain person, object, situation, behavior, etc. can be expressed. Consequently, teaching E/LE students these kinds of speech acts is just as essential. For this reason, the main objective of this article is to explain how an mitigated opinion or assessment can be given in Spanish and how it can be taught in the E/LE classroom. For this, this didactic unit is proposed, which considers that the opinions or evaluations expressed by judges of reality shows such as Operación Triunfo, MasterChef or La voz can be attractive linguistic material to teach how to give an mitigated opinion.

Key words: Opinion. Assessment. Mitigation. Realities Show.

\section{Introducción}

Las opiniones, las valoraciones o los juicios son actos de habla que se realizan en una enorme cantidad de situaciones comunicativas y géneros discursivos, por ejemplo: en una

\footnotetext{
${ }^{1}$ Este artículo ha sido posible gracias al grupo de investigación Val.Es.Co. y al proyecto de investigación FFI2016-75249-P, ES.VAG.ATENUACIÓN ("La atenuación pragmática en su variación genérica: géneros discursivos escritos y orales en el español de España y América"), financiado por el MINECO.

${ }^{2}$ El autor del presente trabajo es beneficiario de una ayuda para la Formación del Profesorado Universitario, financiada por el Ministerio de Educación, Cultura y Deporte de España.
} 
columna de opinión, una entrevista o una conversación coloquial. En muchas ocasiones el hablante atenúa o mitiga dichas opiniones o valoraciones con fines estratégicos. Por ello, consideramos necesario efectuar un sucinto recorrido teórico y aplicado por los dos ejes en torno a los cuales gira el presente artículo y la unidad didáctica que se ha elaborado: la opinión o valoración, por un lado, y la atenuación, por el otro.

\subsection{Enseñar a opinar o valorar en el aula de ELE}

\subsubsection{El acto de habla de opinar, valorar o emitir un juicio}

Establecer una diferencia conceptual entre el acto de opinar, el de valorar y el de emitir un juicio es un problema que no se solucionará en el presente artículo. A efectos de la propuesta didáctica, basta partir de algunas definiciones que pueden ser el soporte teórico de este eje temático.

Desde una perspectiva lexicográfica, si nos remitimos al Diccionario de la Lengua Española (Real Academia Española, en línea), se pueden encontrar las siguientes definiciones: 1) Opinión: «Del lat. opinio, -ōnis. 1. f. Juicio o valoración que se forma una persona respecto de algo o de alguien», 2) Valoración: «1. f. Acción y efecto de valorar $^{3} . »$ y 3) Juicio: «3. m. Acción y efecto de juzgar ${ }^{4} »$. Por otro lado, si partimos de la Teoría de los actos de habla, la opinión es considerada un acto asertivo en el que el/la hablante da a conocer su creencia o punto de vista (opinión) sobre un hecho o una realidad (Searle, 1976: 12). En otro orden, hay algunos autores, como Flores Salgado y Ramírez Cabrera (2015: 91), que consideran que «un juicio o una opinión representan una valoración en la que el hablante evalúa un estado o realidad que puede ir en contra o a favor de lo que cree el interlocutor», de manera que, "esta evaluación pudiese considerarse como un acto dañino para la imagen del oyente».

Aunque estas definiciones no son las únicas que se han vertido, no obstante, para los objetivos que nos hemos propuesto son suficientes, ya que podemos rescatar las siguientes cuestiones: la primera tiene que ver con una posible definición: una opinión puede ser una valoración, un juicio o una evaluación que se forma una persona sobre un estado, una realidad, un objeto, una persona, un ente, etc., en definitiva, respecto de algo o alguien. Y la segunda es que dado que una opinión, una valoración, un juicio o una evaluación se efectúa desde la perspectiva o punto de vista de un hablante, esta puede ir en contra o favor de las creencias, pensamientos o puntos de vista de otros interlocutores. Por tal razón, no es extraño que muchas veces cuando se expresa una opinión, una valoración o un juicio sobre algo o alguien se daña la imagen tanto del hablante como del oyente -sobre todo cuando dicha opinión afecta, más o menos directamente, a este interlocutor (Albelda, Briz, Cestero, Kotwica y Villalba; 2014: 35) -.

\subsubsection{El acto de habla de opinar, valorar o emitir un juicio desde la clase de $\mathbf{E} / \mathrm{LE}$}

El Marco Común Europeo de Referencia para las lenguas (MCER) (Consejo de Europa, 2001) incluye y hace referencia a estos actos de habla. En la presentación de los niveles comunes de referencia (escala global) considera que en el nivel B1 los estudiantes deben

\footnotetext{
${ }^{3}$ Valorar: «De valor. 2. tr. Reconocer, estimar o apreciar el valor o mérito de alguien o algo.»

${ }^{4}$ Juzgar: «Del lat. iudicāre.5. tr. Creer u opinar algo. ¿Juzgas que lo hizo por despecho?»
} 
saber justificar sus opiniones y en el nivel B2 deben defender un punto de vista sobre temas generales. Por otro lado, el cuadro de autoevaluación de los niveles señala, dentro de la competencia de expresión oral del nivel B1, que el estudiante debería explicar y justificar brevemente sus opiniones y proyectos. De la misma manera, señala esto en el nivel B2 (expresión e interacción oral) y en el nivel C1 (interacción oral). Seguidamente, dentro del apartado de las funciones, las nociones, la gramática y el vocabulario que se tienen que desarrollar en cada nivel; el MCER considera que el alumnado, en un nivel $\mathrm{B} 1$, debería ofrecer y pedir «opiniones personales en un debate informal con amigos»; en un nivel B1+ debería ser «capaz de resumir y dar su opinión sobre un cuento, un artículo, un discurso, un debate, una entrevista o un documental»; y en un nivel B2 debería ser «capaz de explicar y defender sus opiniones en un debate, proporcionando explicaciones, argumentos y comentarios adecuados; explica su punto de vista sobre un asunto de actualidad [...]». Por último, cabría destacar que el apartado 4.4. del documento incluye una serie de actividades y estrategias en las que el estudiante tiene que opinar, valorar o emitir juicios en diversas situaciones comunicativas y géneros discursivos como un monologo sostenido (B1), un informe o una redacción (B1), una conversación informal con amigos (B1 y B2), una conversación formal o una reunión de trabajo (B2), entre otros. En definitiva, como se ha podido ver anteriormente, el MCER (Consejo de Europa, 2001) considera que es necesario que el alumnado desarrolle capacidades para expresar opiniones, valoraciones o juicios, sobre todo, a partir de los niveles intermedios (B1-B2).

Por otro lado, el Plan Curricular del Instituto Cervantes (Instituto Cervantes, 2006) incluye un subapartado titulado: «Expresar opiniones, actitudes y conocimientos» dentro del apartado 5. Funciones. En este subapartado se recopilan una serie de estructuras formales que podría emplear el alumnado para pedir o dar una opinión o una valoración, para expresar aprobación o desaprobación, para posicionarse a favor o en contra o para expresar acuerdo o desacuerdo, etc. Cabe mencionar, por una parte, que dichas estructuras se acompañan de ejemplos; y, por otra, que se ofrecen estructuras y ejemplos para todos los niveles. A manera de ejemplo, en el nivel B1, el alumnado podría introducir su opinión mediante las siguientes estructuras: "en mi opinión, desde mi punto de vista, a mí me parece que, yo pienso que, etc.» o, en el nivel B2, el alumnado podría pedir la opinión mediante las siguientes estructuras: «¿Tú qué piensas / opinas + de que + subj.? ¿Qué opinas de que hayan decidido abrir una nueva sucursal tan lejos?, ¿Qué te parece lo de...? ¿Qué te parece lo de las obras en la oficina?, ¿Consideras / Opinas...? ¿Consideras que deberíamos ir?, etc.». En suma, se podría afirmar que el PCIC (Instituto Cervantes, 2006) también considera que es necesario enseñar a opinar, valorar o emitir un juicio.

Finalmente, después de una revisión y análisis de diversos manuales para la enseñanza de $\mathrm{E} / \mathrm{LE}^{5}$, se ha podido ver que la mayoría incluyen unidades o lecciones específicas en las que se abordan dichas funciones: opinar, valorar o emitir un juicio. En concreto, estas unidades y lecciones ofrecen formas o estructuras gramaticales -similares a las que ofrece el PCIC (Instituto Cervantes, 2006)- mediante las cuales se puede, por ejemplo, pedir o expresar una opinión o una valoración. Un aspecto que se debe mencionar es que solicita al alumnado que opine sobre temáticas conflictivas que puedan propiciar la confrontación, el debate o el desacuerdo entre ellos.

\footnotetext{
${ }^{5}$ Los manuales de E/LE analizados son los siguientes: Esespañol. 2. Nivel intermedio. (Alcoba, 2002), Prisma. B1. Progresa. (Vázquez Fernández et alli, 2003), Pasaporte. Nivel 3. B1. (Cerrolaza Aragón et alli, 2008), Abanico. B2. (Chamorro Guerrero et alii, 2010), Bitácora. 3. Nivel B1.1. (Sans Baulenas et alli, 2013), Aula Internacional 3. B1. (Corpas et alli, 2014), Bitácora. 4. B2. (Sans Baulenas et alli, 2017).
} 


\subsection{Enseñar a mitigar o atenuar en el aula de ELE}

\subsubsection{La atenuación: una competencia pragmalingüística}

La atenuación es un fenómeno pragmalingüístico que ha sido ampliamente estudiado en las últimas décadas desde diferentes perspectivas, géneros discursivos, lenguas, etc. El presente artículo, por su parte, no pretende realizar un recorrido bibliográfico por estos trabajos ${ }^{6}$. Ahora bien, sí que considera imperativo, al menos, ofrecer una definición de este fenómeno que pueda ser la base conceptual de la propuesta didáctica:

La atenuación es una actividad argumentativa (retórica) estratégica de minimización de la fuerza ilocutiva y del papel de los participantes en la enunciación para lograr llegar con éxito a la meta prevista, y que es utilizada en contextos situacionales de menos inmediatez o que requieren o se desea presenten menos inmediatez comunicativa. (Briz y Albelda, 2013: 292)

A continuación, se puede ver un ejemplo de una acto de habla de opinión (subrayado) que ha sido atenuado mediante diversos recursos verbales (en cursiva):

(1) Yo creo que ese vestido que te pusiste hoy te queda, pues, un poquito ajustado ¿no?, bueno, esa es mi opinión.

\subsubsection{La atenuación en la clase de $\mathrm{E} / \mathrm{LE}$}

La Enseñanza de Español como Lengua Extrajera (E/LE) es uno de los ámbitos en los que se ha estudiado la atenuación. El apartado 2.3. La atenuación en la didáctica de lenguas extranjeras de la tesis de Secchi (2018) ofrece un recorrido bibliográfico por aquellos trabajos que estudian la atenuación en la enseñanza de E/LE. Asimismo, en la página web del proyecto Es.Vag.Atenuación se recopila una serie de trabajos que versan sobre la atenuación y la intensificación en didácticas de las lenguas.

En lo que respecta al Marco Común Europeo de Referencia para las lenguas (Consejo de Europa, 2001), Negre Parra (2018: 274) señala que «la atenuación no se incluye en el MCER [...]». Así se ha podido comprobar, sin embargo, se podría destacar que el MCER sí que incluye, dentro de la competencia sociolingüística, un subapartado sobre normas de cortesía. En este se explica que debe «evitarse el comportamiento amenazante (dogmatismo, órdenes directas, etc.), expresar arrepentimiento, disculparse por comportamiento amenazante (corrección, contradicción, prohibiciones, etc.)» o bien «utilizar enunciados evasivos, etc. (por ejemplo: «Creo que...» [...]). Aunque no se dice explícitamente que se debe atenuar, sí que se hace de forma implícita.

El Plan Curricular del Instituto Cervantes (Instituto Cervantes, 2006), por otro lado, sí que incluye este fenómeno dentro del capítulo 6.Tácticas y estrategias pragmáticas $\rightarrow$ 3. Conducta interaccional $\rightarrow$ 3.1.Cortesía verbal atenuadora y 3.2.Cortesía verbal valorizante. En concreto, en el subapartado 3.1., se ofrece -para cada nivel- una serie de mecanismos que pueden cumplir una función atenuante. Estos procedimientos se han agrupado según el tipo de atenuación. Esto es: 3.1.1. Atenuación del papel del hablante $o$ del oyente (en todos los niveles), 3.1.2. Atenuación del acto amenazador (en todos los niveles) y 3.1.3. Atenuación dialógica (solo en los niveles: B1, B2, C1 y C2). En el nivel

\footnotetext{
6 Para consultar más trabajos sobre atenuación, puede ver los recopilados por el proyecto Es.Vag.Atenuación en el siguiente enlace: http://esvaratenuacion.es/bibliografiaatenuacion/
} 
B1, por ejemplo, dentro de la atenuación del acto amenazador se sugiere atenuar las opiniones, creencias, afirmaciones mediante verbos performativos, p. ej.: «Creo que debes estudiar más» o «Me parece que no es bueno que comas tantos dulces».

Por último, cabría señalar que el glosario de términos del PCIC define atenuación como:

Estrategia discursiva mediante la cual el hablante minimiza total o parcialmente el contenido proposicional de su enunciado. La atenuación se realiza mediante recursos morfológicos (Es un poco gordo, Está como soso), sintácticos (movimientos concesivos: sí, pero...; periodos condicionales: si acaso no puedes...), etc. En el marco de la cortesía verbal, la atenuación es una estrategia para mitigar la fuerza ilocutiva del acto amenazador para la imagen pública de los intervinientes. Se realiza mediante tácticas como el uso de actos de habla indirectos, desplazamientos en la perspectiva temporal, eufemismos, etc. (Instituto Cervantes, 2006)

En lo relacionado con el tratamiento de la atenuación en manuales, Negre Parra (2018) revisó 7 manuales de enseñanza de ELE, de nivel B2. Los objetivos que se planteó la autora en su estudio fueron: analizar, por una parte, la presencia de recursos y funciones de atenuación y, por otra, determinar qué explotación didáctica de la atenuación hacen los autores del manual. En términos generales, Negre Parra (2018) considera que hay manuales que se adaptan muy bien a las directrices del PCIC (Instituto Cervantes, 2006), pero no obtienen una valoración positiva en cuanto al tratamiento o explotación didáctica del fenómeno. Por el contrario, otros manuales, aunque explotan muy bien didácticamente la atenuación, únicamente desarrollan 4 de los 11 contenidos del PCIC (Instituto Cervantes 2006). Así se puede notar en la Tabla 1.:

\begin{tabular}{|c|c|c|c|c|c|c|c|c|}
\hline MANUAL & $\begin{array}{l}\text { NP PÁGINAS } \\
\text { ANALIZADAS }\end{array}$ & $\underset{\text { (A) }}{\text { EXPLiCITO }}$ & $\begin{array}{c}\text { IMPLíCITO } \\
\text { (B) }\end{array}$ & $\begin{array}{l}\text { EXPLICADO } \\
\text { (C) }\end{array}$ & $\begin{array}{l}\text { USADO } \\
\text { (D) }\end{array}$ & $\begin{array}{c}\text { EXPLOTADO } \\
\text { DIDÁCTICAMENTE } \\
\text { (E) }\end{array}$ & $\begin{array}{c}\text { JUICIO } \\
\text { CRITICO } \\
\text { NEGATIVO } \\
\text { (F) }\end{array}$ & $\begin{array}{c}\text { JIICIO } \\
\text { CRíTICO } \\
\text { POSITIVO } \\
\text { (G) }\end{array}$ \\
\hline$A U L A B 2.1$ & 128 & 7 & 3 & 13 & 7 & 26 & 7 & 39 \\
\hline$A U L A B 2.2$ & 128 & 3 & 6 & 5 & 8 & 13 & 8 & 18 \\
\hline ENCUENTROS B2 & 85 & 6 & 12 & 5 & 9 & 23 & 9 & 28 \\
\hline ES ESPAÑOL 3 & 238 & 7 & 7 & 11 & 25 & 3 & 25 & 14 \\
\hline EXPERTOS & 160 & 5 & 3 & 4 & 6 & 6 & 6 & 10 \\
\hline $\begin{array}{c}\text { ENTORNO } \\
\text { EMPRESARLAL }\end{array}$ & 121 & 0 & 0 & 0 & 16 & 0 & 16 & 0 \\
\hline ENACCION3 & 117 & 14 & 12 & 3 & 7 & 19 & 7 & 22 \\
\hline RESULTADOS: & 977 & 42 & 43 & 41 & 78 & 90 & 78 & 131 \\
\hline
\end{tabular}

Tabla 1. Resultados por manuales (Fuente: Negre Parra [2018:283])

\section{Propuesta didáctica: «Si vas a opinar... no hieras los sentimientos»}

A partir de lo explicado hasta aquí, se ha elaborado la siguiente unidad didáctica titulada: «Si vas a opinar... no hieras los sentimientos». El objetivo principal de la misma es presentar al profesorado de E/LE una propuesta didáctica mediante la cual pueda enseñar a expresar una opinión o una valoración de forma atenuada o suavizada. Para ello, la unidad didáctica se estructura en cinco subapartados: contexto, actividades de exploración de conocimientos previos o precalentamiento, actividades de andamiaje o de incorporación de nuevos saberes, actividades de práctica o de aplicación de nuevos saberes, tarea final y evaluación.

\subsection{Contexto}

La propuesta ha sido diseñada y se puede aplicar tanto para un nivel B1 como para un 
nivel B2. De manera que el docente puede adaptar las actividades y los contenidos al nivel correspondiente. La propuesta, que pertenece a un ámbito público y personal, se ha planificado para llevarse a cabo en cinco sesiones de dos horas de duración. No obstante, el docente también puede prescindir de algunas actividades para ejecutar la propuesta en menos tiempo. Se dirige a estudiantes de ELE en inmersión, aunque también se podría dirigir a otro tipo de estudiantes siempre y cuando el docente contextualice este tipo de programas.

\subsection{Actividades de exploración de conocimientos previos o precalentamiento}

Objetivo I. Reflexionar sobre cómo se opina o se valora en español y sobre cuándo y por qué es necesario suavizar o intensificar nuestras opiniones o valoraciones. Este objetivo se logrará llevando a cabo las siguientes actividades:

2.2.1. Se presenta a los estudiantes una serie de videos en los cuales se vierten opiniones o valoraciones de jueces o jurados de diversos programas de la telerrealidad, por ejemplo: MasterChef, La voz o Got Talent. Se presentan -dependiendo del tiempo que disponga el docente- dos valoraciones: una positiva y otra negativa. Antes del visionado de los videos, se tiene que formular las siguientes preguntas:

a) ¿Qué significa opinar, valorar, evaluar o emitir un juicio? ¿es necesario saber opinar o valorar? ¿por qué?

b) ¿Qué problemas se pueden generar cuando se vierte una opinión o valoración sobre una persona, un comportamiento, un objeto, un tema o una situación?

2.2.2. Luego de ver los diversos videos se formulan las siguientes preguntas:

a) ¿Han visto alguna vez alguno de estos programas de la telerrealidad? ¿cuáles?

b) En estos concursos, ¿qué tipos de opiniones se vierten: positivas o negativas?

c) ¿Qué recursos o procedimientos verbales y no verbales se emplean para intensificar y suavizar las opiniones? y ¿en qué tipo de opiniones se intensifica y en cuáles se suaviza?

d) ¿Por qué y para qué creen que se intensifican o suavizan las opiniones? y ¿Cuáles serían las consecuencias si no se suavizaran las opiniones negativas?

\subsection{Actividades de andamiaje o de incorporación de nuevos conocimientos}

Objetivo II. Explicar a los estudiantes de qué forma se puede pedir y expresar una opinión o valoración; y mediante qué mecanismos verbales y no verbales pueden suavizar o atenuar una opinión. Este objetivo se logrará realizando las siguientes actividades:

2.3.1. Se presenta una nueva opinión negativa vertida por alguno de los jueces de alguno de los programas. Previamente, se pide a los estudiantes que presten atención y anoten según su conocimiento de hablante- aquellos recursos o procedimientos verbales y no verbales que han empleado los jueces para atenuar o suavizar la opinión o la valoración. 
Finalmente, los estudiantes responden las siguientes preguntas:

a) ¿ Mediante qué recursos se atenuó la opinión negativa?

b) ¿Qué habría ocurrido si no se hubiesen empleado estos mecanismos?

c) ¿Mediante qué otros mecanismos se podría atenuar o suavizar esa opinión?

2.3.2. El docente explica, por una parte, qué tipos de atenuadores son los empleados por los jueces, qué se logra al emplearlos y qué función desempeñan. Por otro lado, explica los siguientes aspectos:

a) ¿Mediante qué formas se puede pedir la opinión? Se ofrecen las formas o estructuras que establece el Plan Curricular del Instituto Cervantes (Instituto Cervantes, 2006) para los niveles correspondientes:

\begin{tabular}{|l|l|}
\multicolumn{1}{|c|}{ Nivel B1 } & \multicolumn{1}{c|}{ Nivel B2 } \\
\hline -¿(Tú) qué piensas + de + SN? & - ¿(Tú) qué piensas / opinas + de que + \\
-¿Qué piensas + de que...? & subj.? \\
-¿Te parece que...? & $-i($ Tú) qué piensas / opinas + de lo de \\
- Opinión + ¿Tú qué piensas / crees? / & (que)? \\
¿A ti qué te parece? & -¿Qué te parece lo de...? \\
- En tu opinión / Desde tu punto de & $-¿$ Consideras / Opinas...? \\
vista + O. interrog. & \\
- Según tú + O. interrog. & \\
\hline
\end{tabular}

Tabla 1. Estructuras o formas para pedir una opinión?

b) ¿De qué forma se puede expresar una opinión? Al igual que el inciso anterior, se ofrecen las formas o estructuras que establece el Plan Curricular del Instituto Cervantes (Instituto Cervantes, 2006) para los niveles correspondientes:

\begin{tabular}{|l|l|}
\hline \multicolumn{1}{|c|}{ Nivel B1 } & \multicolumn{1}{c|}{ Nivel B2 } \\
\hline - En mi opinión,... & -A mi modo de ver,... \\
-Desde mi punto de vista,... & -Según... \\
-(A mí) me parece que... & -(Yo) considero que... \\
-(Yo) pienso que... & $-($ Yo) veo que... \\
-(Yo) no creo / no pienso / (A mí) no & $-($ Yo) diría que... \\
me parece que + pres. subj. & -(Yo) no creo / no pienso / no \\
& considero / no opino / no veo / no diría \\
& / (A mí) no me parece que + subj. \\
\hline
\end{tabular}

Tabla 2. Estructuras o formas para expresar una opinión

c) ¿De qué forma o mediante qué mecanismos verbales y no verbales se puede atenuar una opinión? Se ha decidido partir de los recursos o procedimientos que ofrece la Ficha metodológica para el análisis pragmático de la atenuación en corpus discursivos del español (Albelda, Briz, Cestero, Kotwica y Villalba, 2014), aunque también se pueden tomar en cuenta los ofrecidos por el Plan Curricular. En cualquier caso, el docente puede consultar este documento, en el cual se presentan todos los recursos verbales y no verbales atenuantes que recoge la Ficha 
metodológica ${ }^{7}$. Cabe destacar que en este documento se han resaltado en amarillo los mecanismos que podrían ser empleados, desde nuestra perspectiva, en los actos de habla de opinar o valorar -aunque no exclusivamente-. En la Imagen 1. se puede ver un ejemplo de uno de los mecanismos que puede emplearse para atenuar una opinión:

1. Modificadores externos: cuantificadores minimizadores

(15) [Val.Es.Co., G68.B.1.: 391-392]

Al final tuve que hacer unas poquitas más prácticas para sacarme el carné del coche

Imagen 2. Ejemplo de mecanismo para atenuar una opinión o valoración

\subsection{Actividades de práctica o de aplicación de nuevos saberes}

Objetivo III. Emplear mecanismos o recursos -verbales y no verbales- que pueden funcionar como atenuadores de una opinión o valoración. Asimismo, reflexionar sobre la importancia del uso de estos procedimientos atenuantes cuando se opina o valora. Estos objetivos se lograrán realizando las siguientes actividades:

a) Se presenta a los estudiantes dos videos de dos presentaciones realizadas por algunos de los concursantes de los programas de la telerrealidad que se han seleccionado. En este caso, es imperativo que las presentaciones puedan ser valoradas por los estudiantes, de ahí que, es necesario seleccionar una actuación de canto, baile, imitación, etc. Una de las presentaciones debe ser valorada por los jueces del programa de forma positiva y la otra de forma negativa pero atenuada. Es muy importante que los estudiantes solo vean la actuación y no vean las opiniones de los jurados.

b) Después de que los estudiantes hayan visto las actuaciones, deben escribir una breve valoración u opinión de cada una. Se puede ofrecer a los alumnos una hoja con la siguiente estructura:

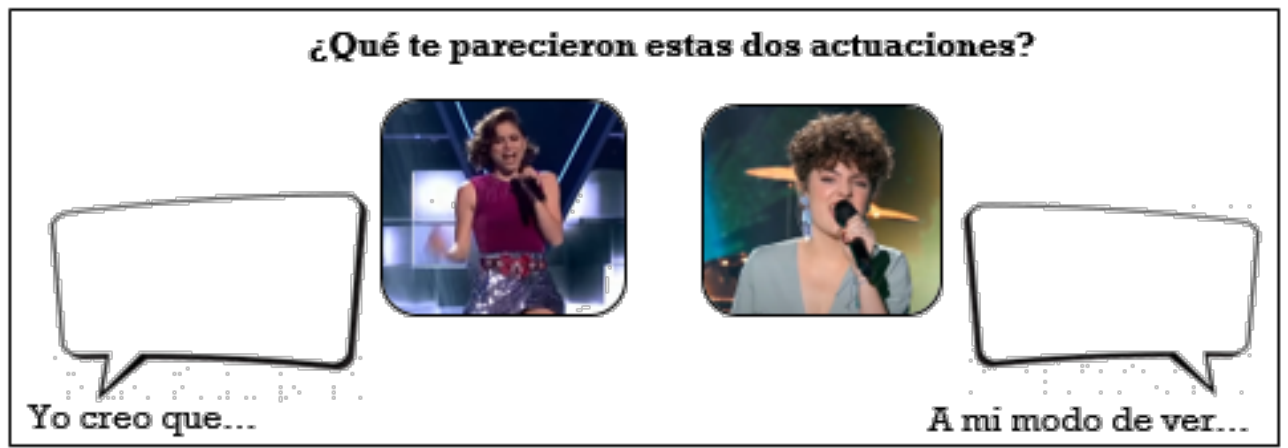

Imagen 2. Ejemplo de estructura del ejercicio para opinar sobre una actuación

\footnotetext{
${ }^{7}$ Es importante resaltar que el docente no tiene por qué explicar cada uno de los mecanismos atenuantes. Podría centrarse, por ejemplo, en aquellos que se han empleado en los videos visualizados y en algunos otros que puedan aparecer en las actividades posteriores.
} 
c) Seguidamente, los estudiantes comparten las opiniones que han escrito sobre las dos actuaciones. El docente puede dirigir esta actividad formulando preguntas como las siguientes:

a) ¿Cuál de las dos actuaciones es mejor, según su punto de vista? ¿por qué?

b) ¿Qué aspectos positivos y negativos consideran que tiene cada una de las presentaciones?

c) ¿Cuál creen que será la valoración u opinión de los jurados para cada una de las actuaciones? ¿positiva o negativa?

d) Luego de compartir las opiniones sobre las presentaciones, los estudiantes escuchan las valoraciones (tanto la positivas como la negativas) que dan los jurados a cada uno de los participantes. Y, posteriormente, se comenta sobre los siguientes aspectos:

a) ¿Quiénes coincidieron con la opinión de los jurados respecto a cada una de las actuaciones? ¿quiénes no? ¿Por qué creen que ocurrió esto?

b) ¿Alguno de los jueces empleó algún recurso atenuante? ¿qué mecanismos empleó y con qué fin? ¿qué tipo de opinión o valoración fue suavizada por los jueces?

c) Respecto a las valoraciones escritas por cada uno, ¿quiénes usaron algún recurso para suavizar su opinión? ¿cuáles? y ¿por qué los emplearon?

e) Finalmente, con el objetivo de observar la importancia del uso de los atenuadores al expresar una opinión, los estudiantes resuelven los siguientes ejercicios ${ }^{8}$ :

1) Encuentra los atenuantes: después de ver los videos de las dos actuaciones anteriores, los estudiantes leen la valoración vertida por los jueces en la que se emplearon recursos atenuantes. Seguidamente, subrayan estos mecanismos y escriben qué tipo de atenuantes son o cuál es la función que desempeñan. Por último, comentan cuáles fueron los recursos empleados.

2) Elimina los atenuantes: Después de haber encontrado todos los atenuantes, los estudiantes eliminan los recursos atenuantes que ha encontrado en las opiniones vertida por los jueces. Para ello, se tendría que reescribir la valoración -solo en caso de ser necesario-. La idea es que, por una parte, puedan leer cuáles han sido específicamente las evaluaciones que han atenuado los jurados y, por otro lado, que reflexionen sobre cuál sería el mensaje que transmitirían las opiniones o valoraciones sin estos recursos; en definitiva, cuál es la importancia de los atenuadores al momento de emitir una opinión-sobre todo, cuando son negativas-.

3) ¡Suaviza nuevamente la opinión!: Ahora que se han eliminado todos los procedimientos atenuantes de la valoración, los estudiantes vuelven a suavizar la opinión; para ello, tienen que sustituir algunos de los mecanismos y recursos atenuantes que empleó el juez por otros equivalentes.

8 Cabe resaltar que estos ejercicios han sido elaborados a partir de las pruebas de análisis para el reconocimiento de la atenuación que propone Villalba (2018) en su artículo Atenuación: algunas claves metodológicas para su análisis. 


\subsection{Tarea final: «Prepárate, actúa y opina»}

Objetivo $I V$. La realización de esta tarea final tiene un doble objetivo: por una parte, preparar y presentar una actuación o un plato de comida como si fuese para un programa de la telerrealidad; $y$, por otra parte, valorar u opinar sobre dichas actuaciones o platillos. Estos objetivos se lograrán realizando las siguientes actividades:

a) El docente, en conjunto con los estudiantes seleccionan alguno de los programas de la telerrealidad que han sido presentados en el aula, por ejemplo: La voz, Operación Triunfo, Got Talent o MasterChef. La selección dependerá del tipo de actuación que los estudiantes deseen preparar y presentar. Es muy importante resaltar que no es obligatorio elegir solo un programa, sino que se puede elegir varios de ellos.

b) Seguidamente, se procede a formar equipos de tres personas ${ }^{9}$, con el objetivo de que cada equipo prepare - dependiendo el programa de la telerrealidad elegidouna actuación de canto, imitación, presentación de un talento (baile, magia, monólogo, gimnasia, etc.) o bien un plato de comida propio de su país.

c) Cada equipo presenta en el aula de clases la actuación o el plato de comida que ha preparado, mientras otro equipo -que será seleccionado por el docente- valora $\mathrm{u}$ opina la actuación o el plato de comida presentado. El docente pedirá a los estudiantes que actúen como jueces: por un lado, que destaquen los aspectos positivos y negativos de la presentación; y, por otro, cuando expresen la valoración negativa, será necesario que empleen algunos recursos y procedimientos atenuantes aprendidos en las sesiones anteriores.

d) Por último, se elige la mejor actuación en cada una de las categorías y se reflexiona sobre la importancia de atenuar las opiniones.

\subsection{Evaluación}

Respecto a la evaluación, en primer lugar, el docente evaluará la expresión oral y comprensión auditiva en las actividades en las que se visualizan y comentan los diferentes videos. En segundo lugar, se evaluará la expresión y comprensión escrita tanto en el ejercicio en el que opinan o valoran dos actuaciones (una positiva y otra negativa) como en el ejercicio en el que tienen que encontrar, eliminar los atenuantes y suavizar nuevamente la opinión. En tercer lugar, en la realización de la tarea final el docente podría evaluar diversos aspectos:

- Preparación y presentación de la actuación o el plato de comida.

- Explicación oral de su presentación o exposición de su plato de comida.

- Expresión oral de las opiniones o valoraciones.

- Uso de procedimientos, mecanismos o recursos atenuantes al expresar las opiniones ${ }^{10}$.

- Uso del léxico específico abordado en las sesiones referente a la comida, el ocio, las actividades artísticas (música, danza, teatro, etc.), entre otros ${ }^{11}$.

\footnotetext{
${ }^{9}$ El número de personas que tendrán cada equipo dependerá de la cantidad de estudiantes

${ }^{10}$ Este aspecto debe evaluarse en los ejercicios anteriores.

${ }^{11}$ El léxico abordado en clase dependerá del tipo de programa seleccionado. De ahí que se pueda tratar léxico referente a la comida (MasterChef) o bien a las actividades artísticas (La voz, Got Talent, etc.)
} 


\section{A modo de conclusión}

Enseñar a un estudiante de E/LE a dar su opinión sobre una determinada temática, a valorar, por ejemplo, un plato de comida o a emitir un juicio sobre el comportamiento que tiene su amigo son solo algunas de las «cosas» que debería saber «hacer» lingüísticamente. Sin lugar a dudas, esta capacidad es esencial, ya que le permite exteriorizar sus puntos de vista respecto de algo o alguien. No obstante, como se ha podido ver en este trabajo, no basta solo con que sepa expresar su opinión, sino que es necesario que sea capaz de atenuar, mitigar o suavizar dichas opiniones o valoraciones mediante recursos verbales y no verbales como las expresiones que las restringen, p. ej.: en mi opinión, a mí parecer, para mí, etc.; diminutivos o, incluso, mediante risas. El alumnado debe ser consciente de que las opiniones o valoraciones, en muchos casos, están cargadas de contenidos negativos, los cuales podrían atentar contra la imagen social de la persona con la que habla.

En definitiva, los estudiantes deberían saber que, para evitar algún conflicto comunicativo al expresar su opinión -especialmente, las de «carga» negativa-, sería más estratégico decir: «No es que me quiera entrometer en tu vida, pero, pienso que la forma de comportarte con tu familia y tu pareja es un poquito injusta, vamos, eso es lo que yo opino» y no: «La forma de comportarte con tu familia y tu pareja es injusta». Esto no significa que siempre será necesario que atenúe sus opiniones, no, sino solo cuando quiera mantener o conservar las relaciones sociales con sus hablantes.

La propuesta didáctica que se ha presentado en este trabajo ofrece una forma de poder enseñar este contenido pragmático, aunque -claramente- no es la única. Desde nuestra perspectiva, las valoraciones $u$ opiniones que vierten los jueces de diversos programas de la telerrealidad como La voz, Operación Triunfo, MasterChef o Got Talent pueden ser muestras de habla útiles para alcanzar los objetivos propuestos: enseñar a opinar de forma atenuada. En estas se puede notar cómo los jurados suavizan sus valoraciones cuando la persona no canta bien una canción y no es aceptada en el programa o cuando el participante cocina y presenta un mal plato de comida. Además, estos programas nos ofrecen otras ventajas, ya que pueden entretener al alumnado, son ampliamente conocidos y se producen en diversos países - muchos de los cuales son hispanohablantes-. Esto último puede ser beneficioso, dado que se podrían trabajar de forma integrada, por ejemplo, las variedades del español.

Ahora bien, este tipo de muestras no son las únicas mediante las cuales se puede enseñar a opinar de forma atenuada, el docente puede acudir a otros géneros discursivos en los que se opine o valore como una tertulia televisiva, una entrevista o encuesta a pie de calle, un debate, una conversación coloquial, comentarios de Facebook o Twitter, reseñas o críticas de películas, reseñas de evaluación de productos y servicios como los que se escriben en foros de opinión o en plataformas de compra como Amazon, Booking, TripAdvisor, entre muchos otros géneros más. Lo importante es que se enseñe a opinar o valorar de forma atenuada. 


\section{Bibliografía}

Albelda MARCO, M. (2010). «¿Cómo se reconoce la atenuación? Una aproximación metodológica». En F. Orletti, y L. Mariottini (eds.); (Des)cortesía en español. Espacios teóricos y metodológicos para su estudio (pp. 47-70). Roma-Estocolmo: Università degli Studi Roma Tre-EDICE.

Albelda, M., Briz, A., Cestero, A. M., Kotwica, D., \& Villalba, C. (2014). «Ficha metodológica para el análisis pragmático de la atenuación en corpus discursivos del español», Oralia, pp. 7-62.

AlcoBA, S. (2002). Esespañol. 2. Nivel intermedio. Madrid: Espasa Calpe.

BRIZ, A., y AlBeldA, M. (2013). «Una propuesta teórica y metodológica para el análisis de la atenuación lingüística en español y portugués. La base de un proyecto en común (ES.POR.ATENUACIÓN)», Onomazein, pp. 288-319.

Chamorro guerrero, M.D., Lozano LóPez, G., MArtínez Gila, P., MuÑoz Álvarez, B., Rosales Varo, F., Ruiz Campillo, J.P. y Ruiz Fajardo, G. (2010). Abanico. B2. Barcelona: Difusión.

CONSEJO DE EUROPA (2001). Marco común europeo de referencia de las lenguas. Instituto Cervantes. Strasbourg: Council of Europe. Para la traducción en español: http://cvc.cervantes.es/obref/marco

Cerrolaza aragón, M., Cerrolaza gili, Ó., Llovet Barquero, B. y Navarro SERRANO, P. (2008). Pasaporte. Nivel 3. B1. Madrid: Eldelsa Grupo Didascalia.

CORPAS, J., GARMENDiA, A. y Soriano, C. (2014). Aula Internacional 3. B1. Barcelona: Difusión.

Flores Salgado, E. \& Ramírez Cabrera, G. (2015). «La atenuación de los actos asertivos: diferencias entre hombres y mujeres», Soprag, 3(1), pp. 90-119.

Instituto Cervantes (2007). Plan Curricular del Instituto Cervantes: niveles de referencia para el español (3 vols.). Madrid: Biblioteca Nueva.

Negre Parra, A. (2018). «Análisis de la atenuación en materiales didácticos de ELE de nivel B2 a la luz del PCIC». Estudios de Lingüistica: ELUA, (4), pp. 269-288.

ReAl ACADEMia EsPañola (en línea). Diccionario de la lengua española, 23. ${ }^{a}$ ed.. Disponible en: https://dle.rae.es [10/04/2020].

Sans Baulenas, N., Martín Peris, E., Garmendia, A. y Conejo, E. (2013). Bitácora. 3. Nivel B1.1. Barcelona: Difusión.

Sans Baulenas, N., Martín Peris, E., Muntal Tarragó, J., Acquaroni Muñoz, R. y CONEJo LóPEZ-L, E. (2017). Bitácora. 4. B2. Barcelona: Difusión.

SeArle, J. (1976). Expression and Meaning: Studies in the Theory of Speech Acts. Cambridge: Cambridge.

SECCHI, D. (2018). La atenuación en aprendices francófonos de E/LE como manifestación de la competencia pragmática. Valencia: Universitat de València.

VÁzQuez Fernández, R., Rollán de CABO, M. Ruiz de Gauna Moreno. M., Blanco Santos, C., Caballero González, G.M., Pardo Díaz, M.I., Reig Sánchez, M., 
Larragaña Domínguez, A., Martín Bosque, A. y Olivar Romero, C. (2003). Prisma. B1. Progresa. Madrid: Edinumen.

VILlALBA IBÁÑEZ, C. (2018). «Atenuación: algunas claves metodológicas para su análisis». Normas, 8, 306-316. Doi: https://doi.org/10.7203/Normas.v8i1.13277 
Apéndice

\section{EJERCICIO 2. OPINAR O VALORAR SIN HERIR LOS SENTIMIENTOS}

Nombre del estudiante:

\section{Encuentra los atenuantes!}

Instrucciones: A continuación lea la valoración que expresa el jueces y subraye los procedimientos, recursos o mecanismos que emplea para atenuar su opinión sobre la actuación de Anne del programa OT.

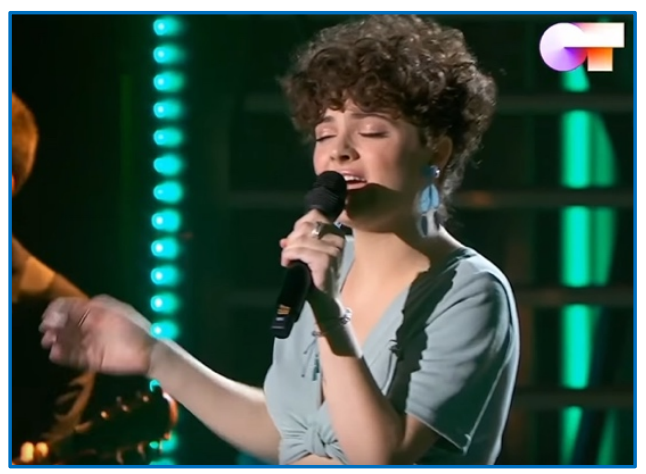

Presentador: ¡Venga, vamos con Portu!

Jurado: sí

Jurado: yo quiero hablar con

Jurado: quiero hablar con Anne.

Presentador: (RISAS)

Participante: perdón.

Jurado: nada.

Jurado: bueno, antes antes de nada sí me gustaría decir que

Jurado: estamos ya casi en la mitad del programa como tú decías

Jurado: y más allá de la valoración artística o técnica que nosotros podamos hacer

Jurado: somos personas que en este lado del programa nos toca ser los malos

Jurado: pero que vemos el canal 24, que seguimos vuestra progresión y que nos encariñamos

Jurado: con lo cual, hacedme caso, esto es doblemente duro ¿vale? para los cuatro.

Jurado: dicho lo cual, vamos con Anne.

Presentador: venga

Jurado: (CHASQUIDO) eehm

Jurado: has estado un poco fastidiada durante toda la semana. Lo sabemos.

Participante: sí

Jurado: has mejorado bastante en la interpretación de hoy

Jurado: pero, no obstante, no sabemos si es suficiente.

Jurado: eh nuestra

Jurado: nuestra óptica es que no has sido convincente del todo

Jurado: en este Looking for paradise ¿no?

Jurado: eeh te ha faltado un punto, Anne. Es lo que sinceramente pensamos.

Jurado: eeh 
Jurado: y lamentablemente te tenemos que proponer para que abandones la academia.

\section{Desaparece los atenuantes!}

Instrucciones: Elimine los procedimientos, mecanismos o recursos atenuantes que ha encontrado en la opinión vertida por el jueces. Para ello, será necesario que reescriba la valoración -en caso de ser necesario-. La idea es que pueda leer cuáles han sido las evaluaciones que ha atenuado el jurado. Muy importante: no es necesario reescribir algunas intervenciones.

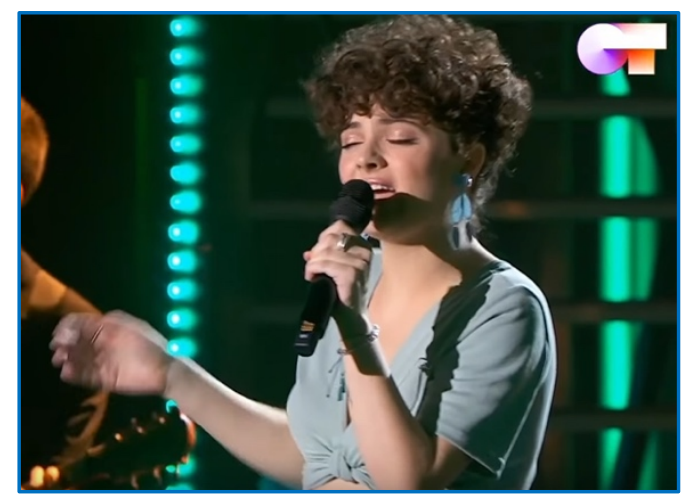

Presentador: ¡Venga, vamos con Portu!

Jurado: sí

Jurado: yo quiero hablar con

Jurado: quiero hablar con Anne.

Presentador: (RISAS)

Participante: perdón.

Jurado: nada.

Jurado: bueno, antes antes de nada sí me gustaría decir que nos toca ser los malos

Jurado: dicho lo cual, vamos con Anne.

Presentador: venga

Jurado: (CHASQUIDO) eehm

Jurado: has estado fastidiada durante toda la semana.

Participante: sí

Jurado: tu interpretación es insuficiente.

Jurado: no has sido convincente.

Jurado: en este Looking for paradise

Jurado: eeh te ha faltado un punto, Anne.

Jurado: eeh

Jurado: y te tenemos que proponer para que abandones la academia.

Presentador: bueno. 


\section{¡Suaviza nuevamente la opinión!}

Instrucciones: Ahora que has eliminado todos los procedimientos atenuantes de la valoración. Vuelve a suavizar la opinión sustituyendo algunos de los mecanismos y recursos atenuantes que empleó el juez por otros equivalentes.

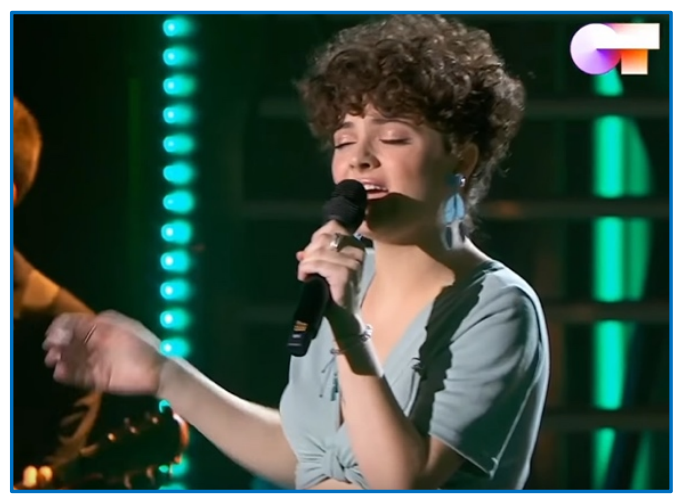

Presentador: ¡Venga, vamos con Portu!

Jurado: sí

Jurado: yo quiero hablar con

Jurado: quiero hablar con Anne.

Presentador: (RISAS)

Participante: perdón.

Jurado: nada.

Jurado: bueno, antes antes de nada sí me gustaría decir que:

nos toca ser los malos

Jurado: pero que vemos el canal 24, que seguimos vuestra progresión y que nos encariñamos.

Jurado: con lo cual, hacedme caso, esto es doblemente duro para los cuatro. Jurado: dicho lo cual, vamos con Anne.

Presentador: venga

Jurado: (CHASQUIDO) eehm

Jurado: has estado fastidiada durante toda la semana. Lo sabemos.

Participante: sí

Jurado: has mejorado bastante en la interpretación de hoy

Jurado: pero, no obstante, si es suficiente.

Jurado: es que no has sido convincente del todo

Jurado: en este Looking for paradise

Jurado: eeh te ha faltado un punto, Anne. Es lo que

Jurado: eeh pensamos.

Jurado: y te tenemos que proponer para que abandones la academia.

Presentador: bueno. 